\title{
Constitutive overexpression of GmDof17-1, a putative DOF transcription factor from soybean causing growth inhibition in tobacco
}

\author{
Jingjing Yu, Guixia Shi, Deyue Yu*
}

Nanjing Agricultural University/National Center for Soybean Improvement, National Key Laboratory of Crop Genetics and Germplasm Enhancement - 210095 - Nanjing - China.

${ }^{*}$ Corresponding author <dyyu@njau.edu.cn>

Edited by: Daniel Scherer de Moura

Received December 06, 2012

Accepted September 13, 2013

\begin{abstract}
The Dof proteins belong to a large family of plant transcription factors that share a single highly conserved zinc finger and play an important role in many physiological processes. To elucidate the function of Dof in soybean, GmDof17-1 was cloned from soybean (Glycine max (L.) Merr); the open reading frame (ORF) of GmDof1 7-1 is $846 \mathrm{bp}$ and encodes a putative protein that includes 281 amino acids. Using qRT-PCR, the expression profiles of GmDof17-1 were obtained for various parts of the soybean plant. GmDof17-1 was primarily expressed in the roots and pods at various stages of pod development. The gene was ectopically expressed in tobacco under the control of the $2 \times$ CaMV35S promoter to study the functions of the gene product. The transgenic tobacco plants showed to be dwarf, and the indole-3-acetic acid (IAA) content was decreased, whereas the sulfur-containing amino acid content of the seeds increased. These results provide new insight into the function of GmDof1 7-1 in seed development.
\end{abstract}

\section{Introduction}

Dof proteins are a major family of plant transcription factors that have a strongly conserved DNA-binding domain, which contains a single $\mathrm{C}_{2} \mathrm{C}_{2}$-type zinc-fingerlike motif that is generally located in the $\mathrm{N}$-terminal region and a C-terminal domain for transcriptional regulation (this region has divergent amino acid sequences) (Yanagisawa and Izui, 1993; Yanagisawa, 2004). Since Dof1 (the first identified Dof protein) was found in maize (Yanagisawa and Izui, 1993), many Dof proteins have been discovered, which participate in specific biological processes in other plants. The functions of reported Dof genes are highly diverse and include roles in hormone response, defense response, organ development and other responses (Baumann et al., 1999; Kang and Singh, 2001; Kang et al., 2000; 2003; Kisu et al., 1998; Mena et al., 1998; Plesch et al., 2001; Vicente-Carbajosa et al., 1997; Wang et al., 2007; Ward et al., 2005; Washio, 2001; Xin et al., 2009; Yang et al., 2010).

Phytohormones play important roles in coordinating many growth-related processes throughout the plant life cycle, including embryogenesis, vascular differentiation, organogenesis, tropic growth and root and shoot architecture (Kepinski, 2006; Quint and Gray, 2006; Shan et al., 2012 Stamm and Kumar, 2010). Some researchers have studied the interactions between Dof proteins and phytohormones and the tobacco Dof protein NtBBF1 has been found to be involved in the auxin-inducible expression of rolB (Baumann et al., 1999).

The expression of the pumpkin ascorbate oxidase gene (AOBP), which binds to the AGTA repeat through the Dof domain, is regulated by auxin (Kisu et al., 1998; Esaka et al., 1992). PtDof1 was isolated from Populus tomentosa, which exhibits tissue-specific expression (Xin et al., 2009). These studies indicate possible roles for Dof proteins in the auxin response. In Arabidopsis, OBP3 (AtDof3.6) was induced by salicylic acid (Kang et al., 2003), and OBP3 is a component in phytochrome and cryptochrome signaling. OBP3 mutants exhibit a root defect and yellowish leaves (Ward et al., 2005). The expression of OBP3-responsive genes (ORGs) is up-regulated by SA but down-regulated by jasmonic acid (JA).

Wang et al. (2007) identified 28 Dof-type transcription factor genes in soybean. Two genes, GmDof4 and $G m D o f 11$, were found to increase the content of total fatty acids and lipids in transgenic Arabidopsis seeds. Aiming to further study the function of Dof-type genes, we focused on GmDof17. We found that GmDof17 exists as two copies in the soybean genome, which are located on chromosomes 7 and 16, and are tentatively named GmDof17-1 and GmDof17-2, respectively. We cloned GmDof $17-1$ and investigated its role in transgenic tobacco.

\section{Materials and Methods}

\section{Plant Material and Growth Conditions}

Soybean seeds [Glycine max (L) Merr]. cv. Nannong 94-16 were cultivated in the field under natural conditions in Nanjing, China $\left(32^{\circ} 03^{\prime} \mathrm{N} ; 118^{\circ} 46^{\prime} \mathrm{E}\right)$. The roots, stems, leaves, flowers and seeds were harvested at various stages, frozen in liquid nitrogen and stored at $80^{\circ} \mathrm{C}$ for further analysis. Transgenic tobacco (Nicotiana tabacum cv. SamSun) plants and non-transgenic wild type (WT) controls were grown at $25 / 22{ }^{\circ} \mathrm{C}$ under a 16/8-h light/dark cycle using artificial light. Transgenic and WT tobacco plants at similar developmental stages were used in this study. For indole-3-acetic acid (IAA) treatments, the seedlings were sprayed with $1 \mathrm{mg} \mathrm{L}^{-1}$ IAA. Leaves from the treated seedlings and from the control plants were sampled at 0,2 and $8 \mathrm{~h}$ after treatment and immediately frozen in liquid nitrogen for RNA extraction.

\section{Phylogenetic analysis}

The sequences of the Dof domains from soybean and other plants were compiled and aligned using 
Clustal X to obtain the optimal trees. The phylogenetic tree was constructed using MEGA4 (Kumar et al., 2008) using neighbor-joining and 1,000 bootstrap replicates. Previously published plant Dof transporter-like gene sequences were retrieved from the NCBI database with their corresponding protein numbers.

\section{DNA and RNA extraction and first-strand cDNA synthesis}

Genomic DNA was extracted using the CTAB (Cetyltrimethyl Ammonium Bromide) protocol with minor modifications (Weising et al., 1995). To analyze the expression of the GmDof17-1 gene in the transgenic tobacco plants and wild type plants, RNA was extracted for quantitative RT-PCR (Real time-polymerase chain reaction). Total RNA was isolated from the roots, stems, leaves, flowers and pods of the soybean cultivar "Nannong 94-16" harvested at various stages (20 DAF (Days after flowering), 30 DAF, 40 DAF and 50 DAF) using Trizol reagent (Invitrogen; Carlsbad, CA) according to the manufacturer's protocol. RNA was sequentially treated with DNase I (Promega; Madison, WI) at $37{ }^{\circ} \mathrm{C}$ for 15 min to remove the remaining genomic DNA. First-strand cDNA was synthesized with $2 \mu$ of purified RNA using the First-Strand cDNA Synthesis Kit (TaKaRa, Japan) according to the manufacturer's protocol. The soybean $a c$ tin gene (GenBank accession no. V00450) was amplified and used as the internal control in these experiments. All reactions were performed in triplicate.

\section{Quantitative RT-PCR (qRT-PCR)}

To analyze the expression of the GmDof17-1 gene in soybean, total RNA was extracted for quantitative RT-PCR using the TRIZOL reagent (Tiangen, Beijing) in accordance with the manufacturer's protocol and digested using RNase-free DNaseI (Promega) at $37{ }^{\circ} \mathrm{C}$ for $15 \mathrm{~min}$.

PCR amplification was performed on an MJ Research thermocycler using a DyNAmo SYBR Green qRTPCR kit (Finnzymes) according to the manufacturer's protocol. Each reaction was performed on $2 \mu \mathrm{L}$ of diluted cDNA in a total reaction volume of $10 \mu \mathrm{L}$. The reaction procedure was set according to the manufacturer's protocol, and the primer sequences were $5^{\prime}$-TTA TTA CAA CAA CTA CAG CCT CAC ACA-3' and 5'-GAT GAG AGG TTA ATT GGT GGG TTT-3'.

For each quantitative RT-PCR experiment, at least three duplicates of each sample and three processing times were performed. The relative expression level of each gene was calculated using the $2^{-\Delta \Delta t}$ method (Livak and Schmittgen, 2001).

\section{Vector construction and transformation}

The full-length sequence of GmDof17-1 was obtained using PCR. The PCR products were purified from low melting point agarose gels using a Promega Wizard PCR purification kit, and the DNA fragment was eluted in TE buffer ( $\mathrm{pH}$ 8.0). The expression vector was con- structed using Gateway Technology with the Clonase ${ }^{\mathrm{TM}}$ II kit (Invitrogen) (Chen et al., 2010). The pMDC83GmDof17-1 vector was introduced into the Agrobacterium tumefaciens strain EHA105 using the freeze-thaw method. Nicotiana tabacum cv. SamSun was transformed using the leaf disk method (Hoekema et al., 1983). The transgenic tobacco plants were regenerated from transformed leaf disks following selection on $50 \mathrm{mg} \mathrm{L}^{-1}$ hygromycin. The transgenic plants were identified using PCR and qRT-PCR.

\section{Subcellular localization of GmDof 17-1}

Unlike the sub-cellular localization of the proteins in onion epidermal cells, the leaves of transgenic tobacco plants were collected for GFP fluorescence detection. The epidermal cells from the leaves were laid flat on a glass slide and a drop of distilled water was added to the center of the slide, and the sample was covered with a coverslip. Protein expression was observed under a confocal microscope.

\section{IAA determination}

Fresh WT leaves and leaves from transgenic plant lines 4 and 7 were frozen at $70{ }^{\circ} \mathrm{C}$ and finely pulverized in liquid nitrogen. The weighed leaf powder was suspended in $4 \mathrm{ml}$ of $80 \%$ methanol that included $0.3 \mathrm{mmol}$ $\mathrm{L}^{-1}$ sodium diethyl dithiocarbamate (Sigma) and was incubated at $20{ }^{\circ} \mathrm{C}$ overnight. The samples were centrifuged for $10 \mathrm{~min}$ at $1,000 \mathrm{~g}$ at $4{ }^{\circ} \mathrm{C}$, and the supernatants were collected (AVANTI J-251, Eppendorf; Germany). This procedure was repeated twice. The supernatants were mixed and analyzed using high performance liquid chromatography (PROSTAR-230, Varian Inc.; USA). The data were statistically analyzed using the Student's t test.

\section{Analysis of IAA treatment in transgenic tobacco plants}

Surface-sterilized seeds of WT and T1 transgenic GmDof17-1 tobacco plants were germinated on MS medium for 15 days and the resulting seedlings transferred to MS medium containing various IAA concentrations 10 and $\left.1 \mathrm{mg} \mathrm{L}^{-1}\right)$ for ten days. The IAA concentration used was selected in accordance with the literature (Casimiro et al., 2001; 2003).

\section{Results}

\section{Isolation and characterization of the GmDof17-1 gene in soybean}

GmDof17 exists as two copies in the soybean genome, which are designated GmDof17-1 and GmDof17-2. GmDof17-1 (Genbank accession number ABI16018) contains a complete open reading frame (ORF) of $846 \mathrm{bp}$ and encodes a putative protein of 281 amino acids with a predicted molecular mass of $30.64 \mathrm{kDa}$ and a predicted isoelectric point (pI) of 9.00. The GmDof17-2 gene encodes a protein with 276 amino acid residues (Figure 1). 


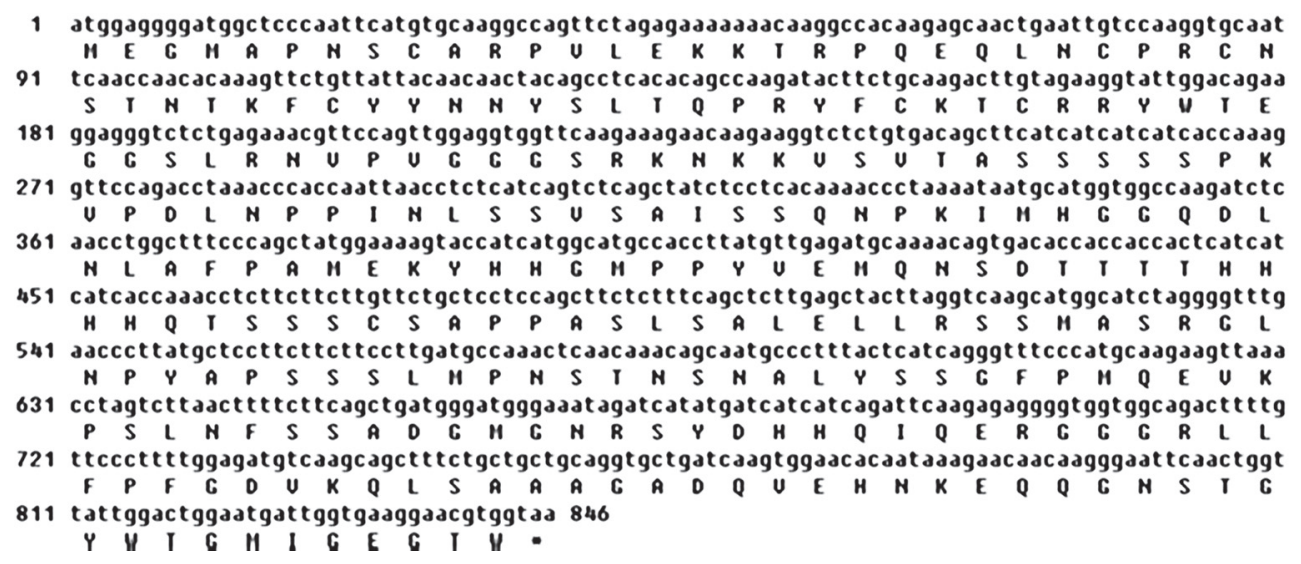

Figure 1 - Nucleotide and amino acid sequences of the GmDof1 1-1 gene and translated protein in soybean.

A conserved domain search in NCBI showed that GmDof17-1 includes a complete Dof domain (http://www. ncbi.nlm.nih.gov/). The Dof domain and four cysteine residues are putatively involved in the formation of the zinc finger. It has been confirmed that GmDof17 is nuclear protein with transcriptional activation activity (Wang et al., 2007), and we found that GmDof17-1 is localized in the nucleus in overexpressed transgenic tobacco line cells under control of the $2 \times$ CaMV35S promoter (Figure 2).

\section{Expression of GmDof17-1 in various soybean or-} gans

GmDof17-1 was expressed in all of these organs, and the abundance of GmDof17-1 mRNA was higher in roots, flowers and pods than in stems and leaves (Figure 3 ). The localization of expression suggests potential roles for GmDof17-1 in root and seed development.

\section{Alignment and phylogenetic analysis}

Alignment of the deduced amino acid sequence of GmDof17-1 and other reported homologous Dof proteins shows that GmDof17-1 shares a highly conserved zinc finger motif in the N-terminal region that could mediate DNA binding or protein-protein interactions (Yanagisawa, 2002); other residues located in the C-terminal transcriptional activation regions were different (Figure 4). This implies that these proteins participate in diverse biological functions.

GmDof17-1 was clustered with DAG1 and DAG2, which play an important role in the germination of Arabidopsis seeds (Figure 5), and with GmDof17-1, 19, 21 and 28 , which is consistent with a previous study (Wang et al., 2007).

Overexpression of $\mathrm{GmDof17-1}$ inhibits transgenic tobacco plant growth

The height and root length of the transgenic tobacco plants (Figure 6) were considerably shorter than in wild type plants $(p>0.05)$ (Figure 7) and some seeds even failed to germinate (data not shown). These results indicate that GmDof17-1 inhibits plant growth.

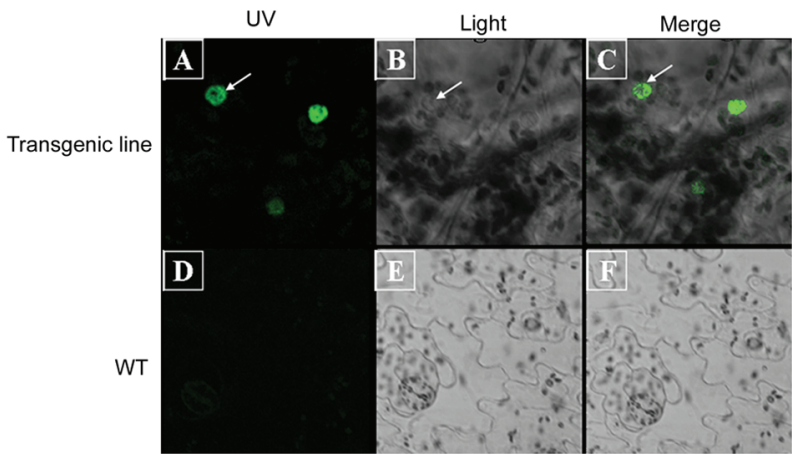

Figure 2 - Subcellular localization of GmDof17 in overexpressed transgenic tobacco cells. A. Dark field images show green fluorescence in the transgenic tobacco cells. B. Bright field images show the morphology in transgenic tobacco cells. C. Merged images of transgenic tobacco cells. D. Dark field images show green fluorescence in wild type tobacco cells. E. Bright field images show the morphology in wild type tobacco cells. F. Merged images of wild type tobacco cells.

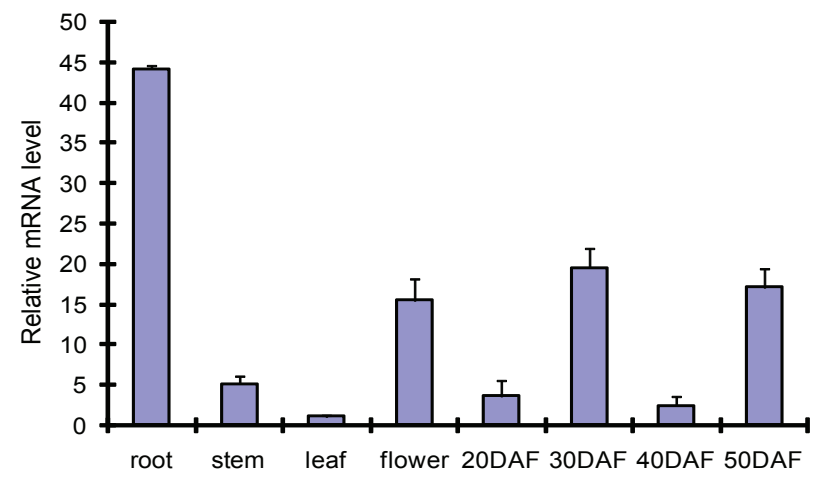

Figure 3 - Analysis of GmDof17-1 expression using quantitative RT$\mathrm{PCR}$ in roots, stems, leaves, flowers and pods at various growth stages (20 days after flowering- DAF-, $30 \mathrm{DAF}, 40 \mathrm{DAF}$ and 50 DAF). 

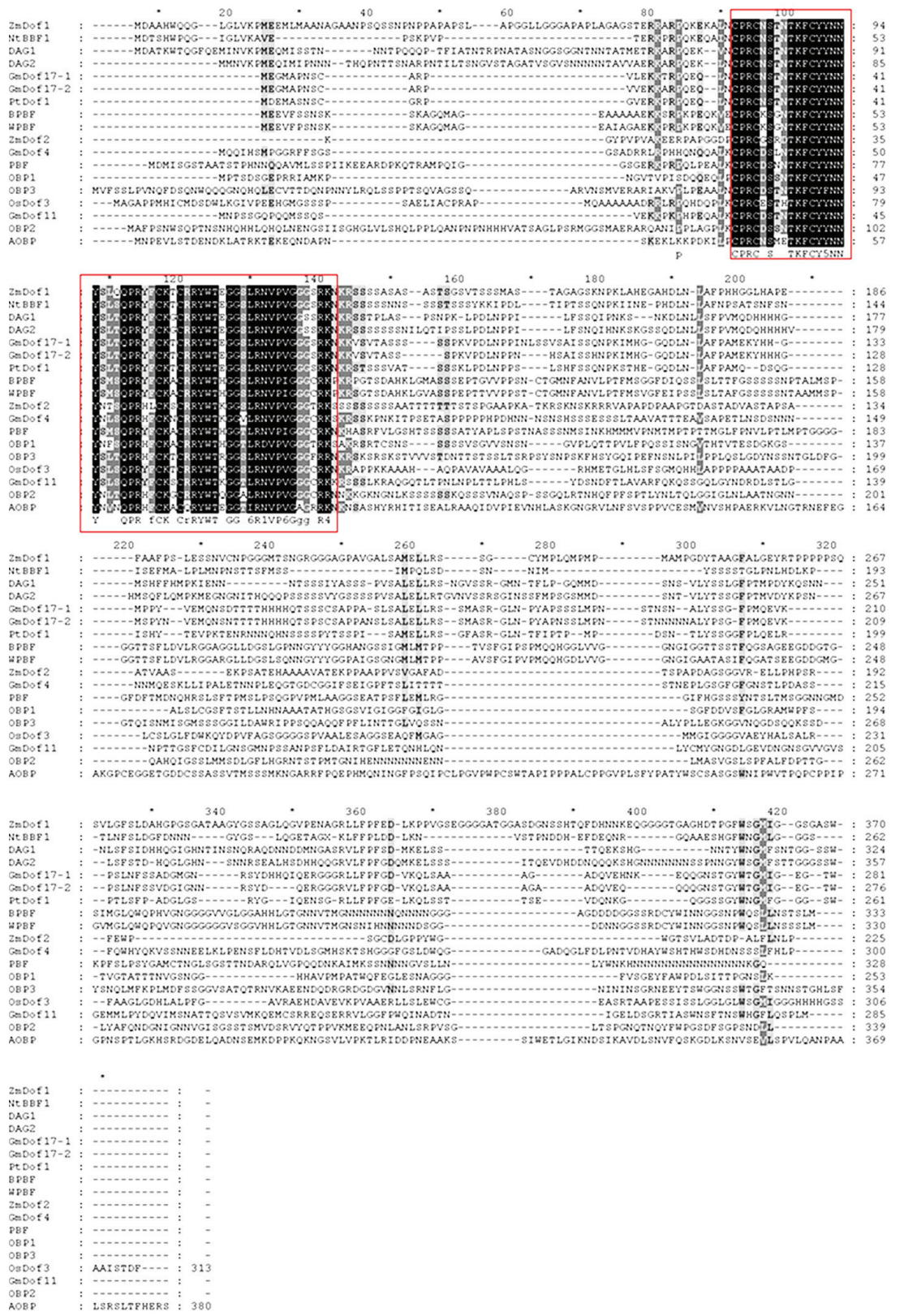

Figure 4 - Sequence of 18 other Dof-type proteins. Protein accession numbers: ZmDof1: NP_001150043; ZmDof2: CAA56287; PBF: NP_001105400; BPBF: BAK06363; WPBF: CAA09976; OBP1: NP_190610; OBP2: NP_001030988; OBP3: NP_974442; DAG1: NP_001118885; DAG2: AAP68281; NtBBF1: CAA08755; OsDof3: AAL87148; GmDof4: NP_001236530; GmDof11: NP_001236626; PtDof1: XP_002320208; AOBP: BAA08094; GmDof17-1: NP_001236720; and GmDof17-2: XP_003548410.

Also, the endogenic IAA content of transgenic tobacco plants was found to be significantly less than that in WT plants (Figure 8).

\section{Exogenous auxin promotes the growth of transgenic tobacco plants}

Plant height, root length and fresh weight of transgenic plants reached the same levels as those of WT plants $(p>0.05)$ (Figure 9). Exogenous auxin can promote the growth of transgenic tobacco plants. These results demonstrate that the dwarfism of the transgenic plants results from a lack of endogenic IAA.

\section{Modification of soybean protein quality}

The GmDof17-1 protein affected the growth of the 


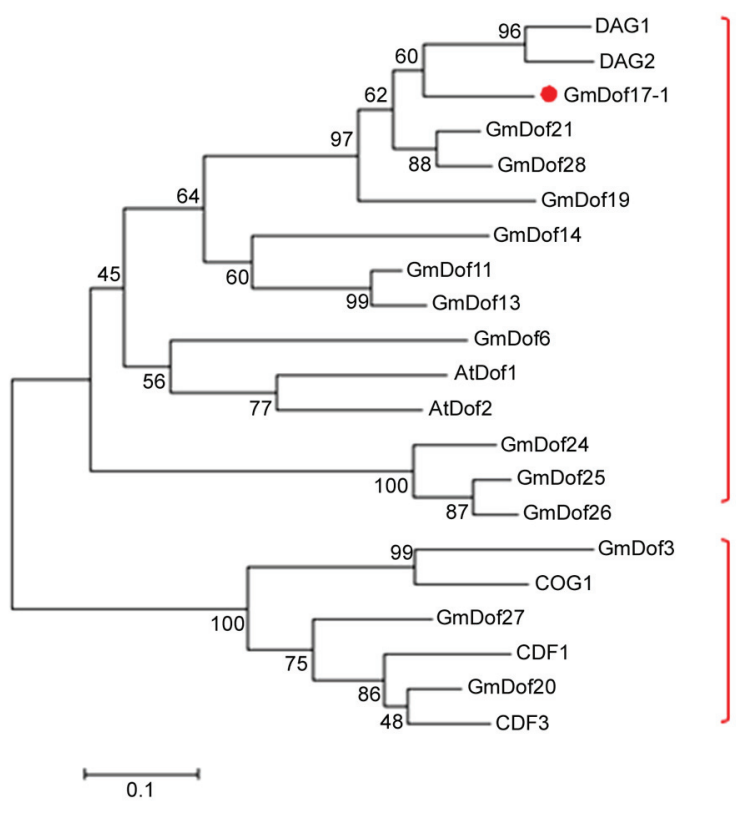

Figure 5 - A phylogenetic tree based on the alignment of the soybean GmDof17-1 gene with 19 other Dof-type proteins. The tree was constructed using MEGA5 with neighbor-joining and 1,000 bootstrap replicates. The numbers indicate percentage bootstrap support. Protein accession numbers: GmDof3 (Glyma13g30331), GmDof6 (Glyma16g26030), GmDof11 (Glyma13g40420), GmDof13 (Glyma11g15761), GmDof14 (Glyma11g15761), GmDof17-1 (Glyma07g05950), GmDof19 (Glyma20g35910), GmDof20 (Glyma17g10920), GmDof21 (Glyma04g41170), GmDof24 (Glyma07g31340), GmDof25 (Glyma15g07730), GmDof26 (Glyma13g31560), GmDof27 (Glyma19g02710), GmDof28 (Glyma06g13671), COG1 (At1g29160), AtDof1 (At1g51700), AtDof2 (At3g21270), DAG1 (At3g61850), DAG2 (At2g46590), CDF1 (At5g62430), and CDF3 (AT2G38530).

plant as well as increasing the methionine and cysteine contents.. The sulfur-containing amino acid content was higher in the transgenic lines than in WT (up to 28 $\%$ higher) (Figure 10). Moreover, the methionine and cysteine contents were higher by $30 \%$ and $9.4 \%$, respectively (Figure 10). GmDof17-1 gene expression might be related to an increase in the sulfur-containing amino acid content. Our study lays the foundation for the transformation of quality and nutritional improvement of soybeans using transgenic technology (Yu et al., 2012).

\section{Discussion}

Dof proteins, which function as transcriptional activators or repressors, are involved in diverse plant-specific biological processes. Dof domains play critical roles in plant growth and development, although their physiological roles remain unknown (Yanagisawa, 2004). In this report, we described the characterization of GmDof17-1 protein that belongs to the zinc finger family. As a puta-

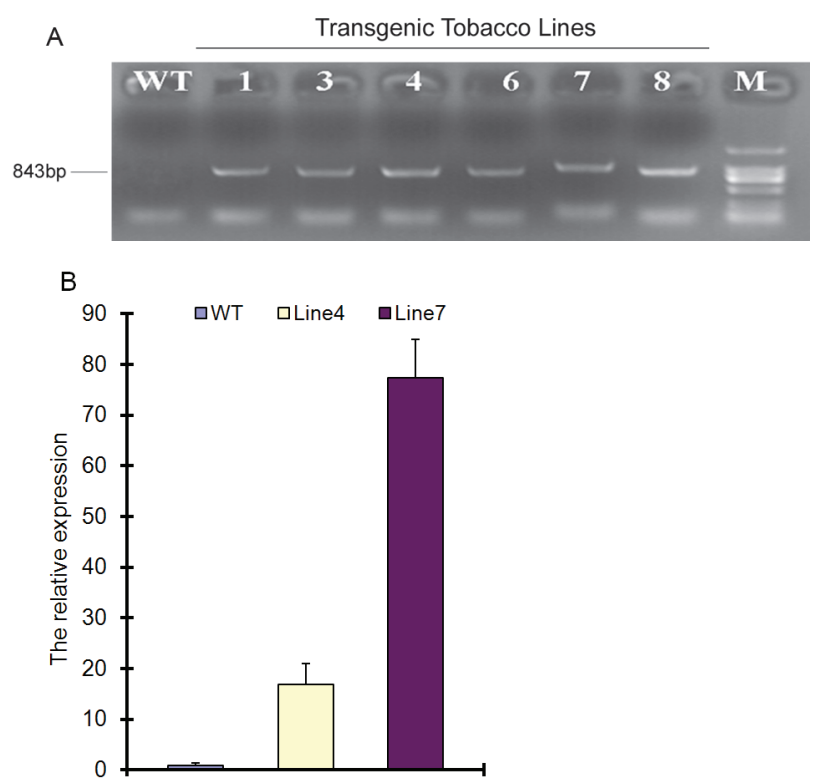

Figure 6 - Identification of GmDof17-1-overexpressed transgenic lines and WT tobacco plants using PCR and qRT-PCR. A. The PCR products of the transgenic lines; B. qRT-PCR of the transgenic lines.

tive transcription factor, GmDof17-1 plays an important role in the development of roots, flowers, seeds and sulfur-containing amino acid content by regulating auxin.

Tian et al. (2004) identified 39 putative unigenes that encode Dof-like proteins in soybean by analyzing their ESTs (Expressed sequence tags). Wang et al. (2007) found 28 Dof genes in soybean based on their highly conserved Dof domains, and it was suggested that GmDof4 and GmDof11 increase the lipid content of seeds by activating genes that are associated with lipid biosynthesis and suppress the expression of storage protein genes.

In the GmDof17-1 transgenic plants, an increase $(26 \%)$ in the sulfur-containing amino acid content was observed; however, no change in the lipid content was found (data not shown). The content of various amino acids increased in the transgenic lines, such as the sulfur-containing amino acids (methionine and cysteine) and proline (Figure 11). The sulfur-containing amino acid content affects the nutritional value of proteins. The main methods used to increase the sulfur-containing amino acid content of soybean are traditional molecular breeding and genetic engineering.

Many authors have noted the relationships between Dof transcription factors and hormone signaling, such as NtBBF1 and auxin in tobacco, AOBP and auxin in pumpkin and OBP3 and salicylic acid in Arabidopsis (Baumann et al., 1999; De Paolis et al., 1996; Kang and Singh, 2001; Kang et al., 2000; 2003; Kisu et al., 1998; Ward et al., 2005). In Arabidopsis thaliana, the Dof protein DAG1 mediates PIL5 activity during seed germination by negatively regulating the GA biosynthetic gene (Gabriele et al., 2010). Our study demonstrates that the 

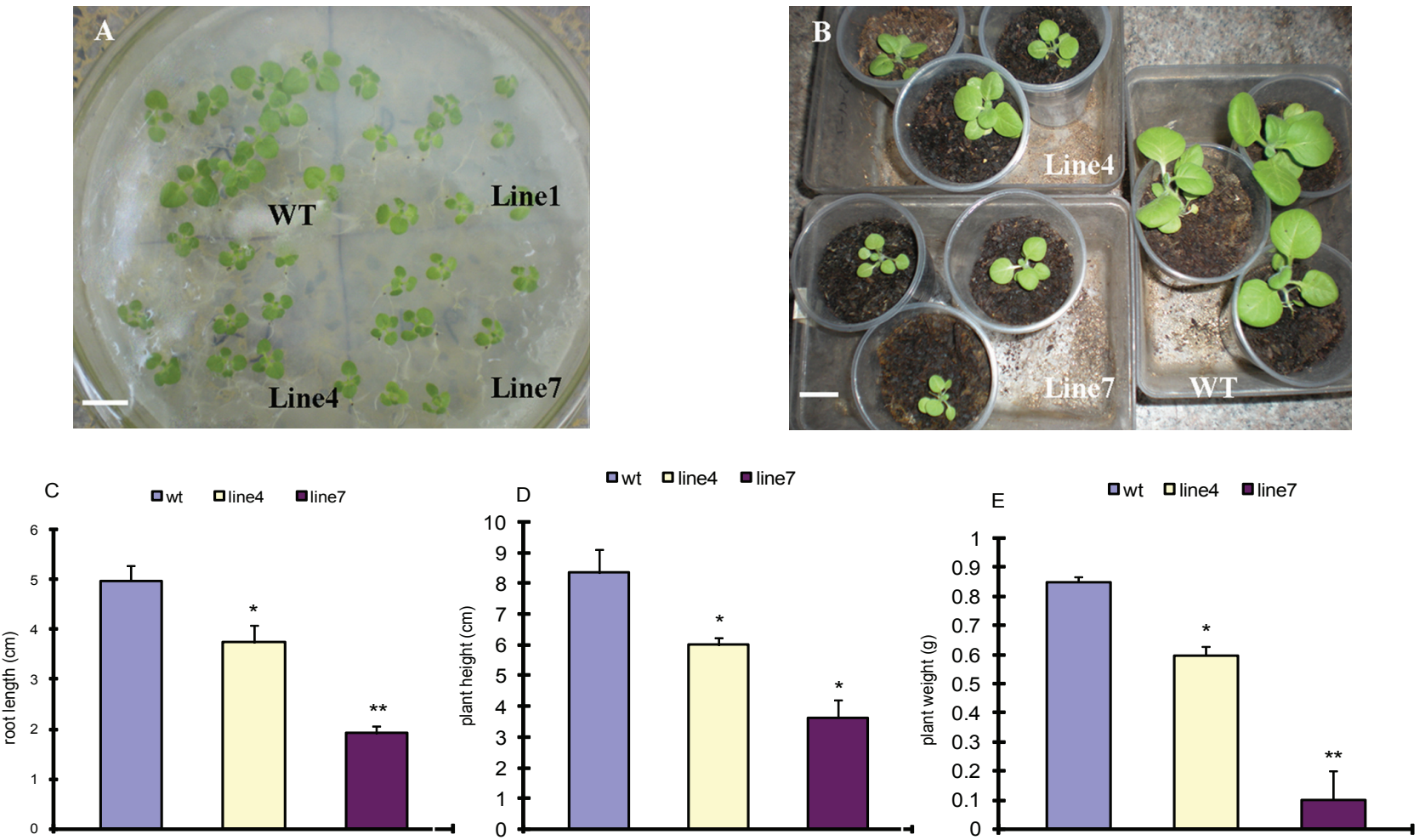

Figure 7 - The development of wild type and GmDof1 7-1 T1 transgenic tobacco lines. A. Wild type and transgenic tobacco plants in MS medium; the bar represents $2 \mathrm{~cm}$. B. The wild type and transgenic tobacco plants were moved to healthy soil; the bar represents $5 \mathrm{~cm}$. $C$. Root lengths without IAA treatment. D. Plant heights without IAA treatment. E. Plant weights without IAA treatment. ${ }^{*}$ Significant at $p<0.05$; ${ }^{\star *}$ Significant at $p<0.01$.

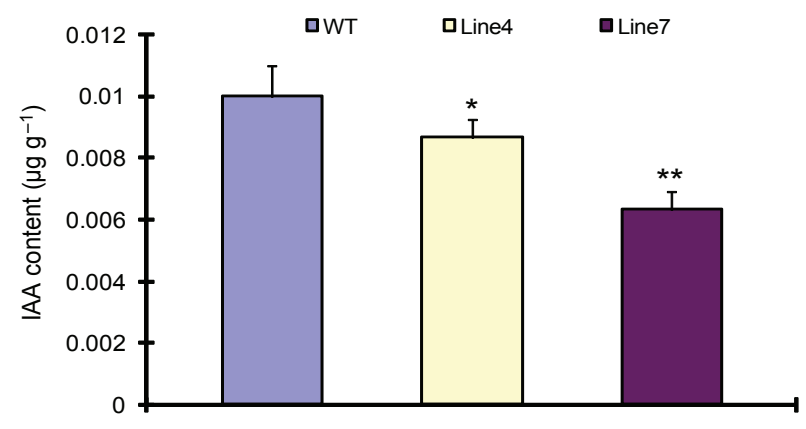

Figure 8 - IAA content in wild type and GmDof17-1 T1 transgenic tobacco lines. * Significant at $p<0.05$; * ${ }^{*}$ Significant at $p<$ 0.01 .

ectopic overexpression of GmDof17-1 in tobacco reduces plant height and the endogenous auxin content, whereas exogenous auxin compensates for the lack of endogenous auxin content. Based on the clustering of GmDof17-1 with DAG1 and DAG2 in the phylogenetic tree, the functions of GmDof17-1 and DAG might have some similarities.

Sulfur-containing amino acids (Met and Cys) are important for plant growth, and their metabolism has been implicated in plant growth through the plantgrowth hormones cytokinin and auxin (Ravanel et al., 1998). Alterations were observed in the contents of sul- fur-containing metabolites in response to the manipulation of the expression of Aux/IAA and ARF transcription factors, especially in the key metabolites cysteine and glutathione (Falkenberg et al., 2008). The relationship between the increased sulfur-containing amino acid content and decreased auxin content found in our research needs further study.

Models describing signal processing and plant development are now shifting from a set of linear and separate transduction pathways to network-level structural formation (Genoud and Metraux, 1999; Gutierrez et al., 2007). GmDof17-1 is a member of the Dof family of transcription factors, which play roles in plant development and growth, and the Dof family requires further characterization, especially with regard to its role in auxin.

\section{References}

Baumann, K.; De Paolis, A.; Costantino, P.; Gualberti, G. 1999. The DNA binding site of the Dof protein NtBBF1 is essential for tissue-specific and auxin-regulated expression of the rolB oncogene in plants. The Plant Cell 11: 323-333.

Casimiro, I.; Beeckman, T.; Graham, N.; Bhalerao, R.; Zhang, H.; Casero, P.; Sandberg, G.; Bennett, M.J. 2003. Dissecting Arabidopsis lateral root development. Trends in Plant Science 8: $165-171$. 

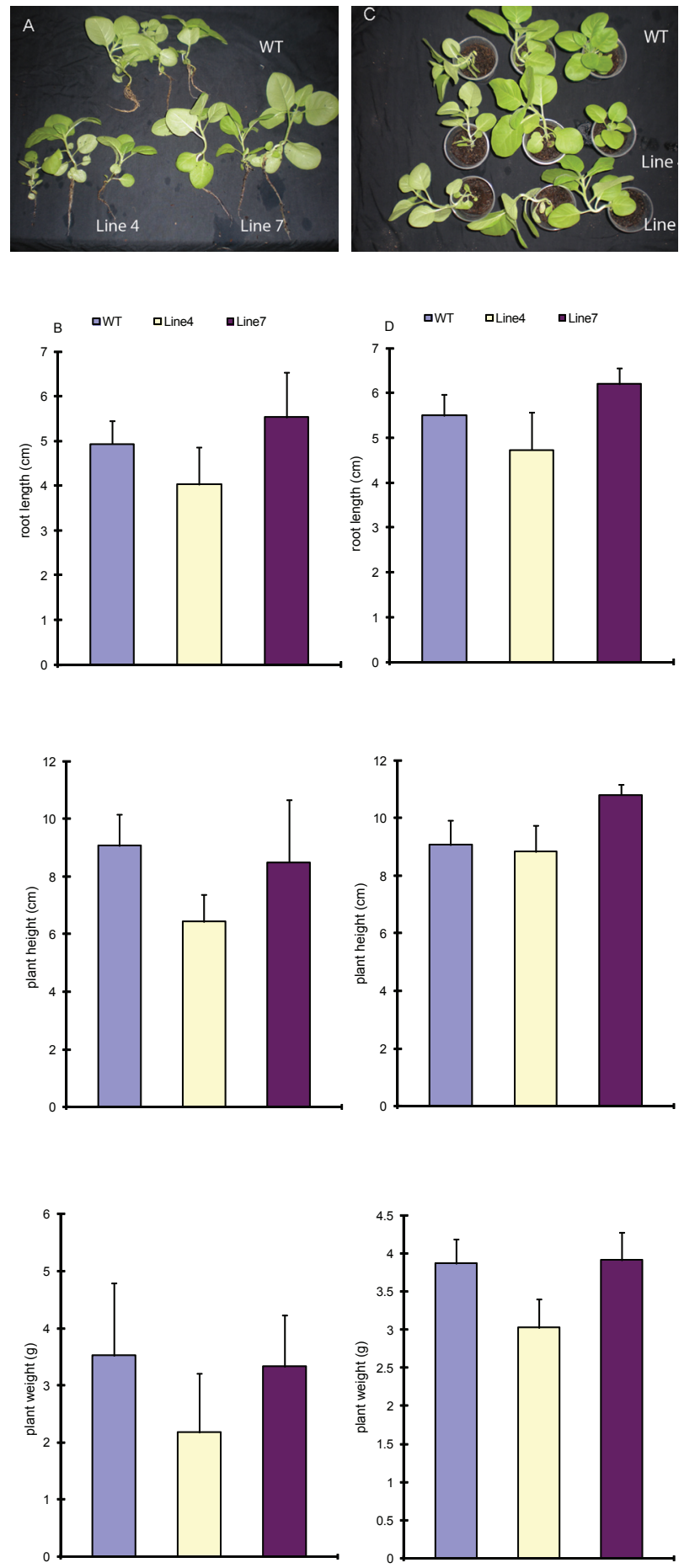

Figure 9 - Phenotype analysis of GmDof1 7-1 T1 transgenic tobacco lines after $1 \mathrm{mg} / \mathrm{L} I A A$ treatment. A. The phenotype of wild type and GmDof17-1 T1 transgenic tobacco lines after IAA treatment. B. Root length, plant height and fresh weight measurements after IAA treatment. C. Another group showing the phenotype of wild type and GmDof1 7-1 T1 transgenic tobacco lines after IAA treatment. D. Another group showing root length, plant height and fresh weight measurements after IAA treatment.
aWT $\quad$ transgenicline

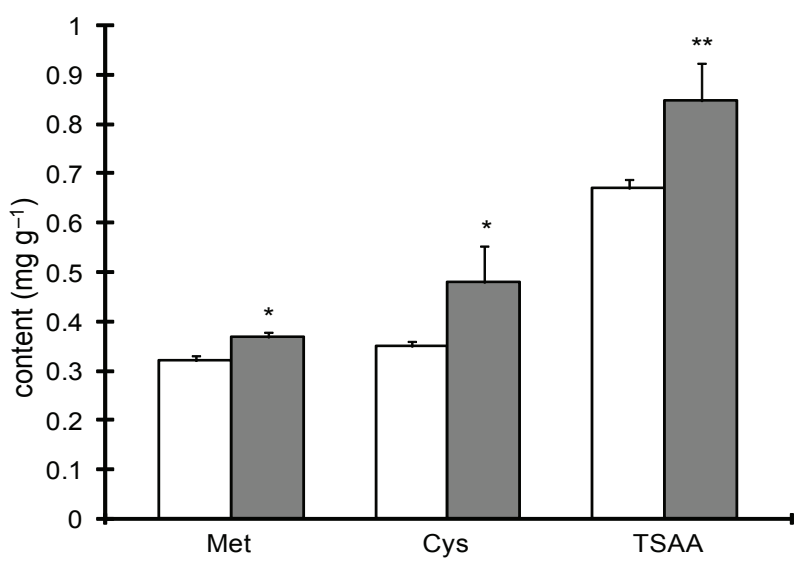

Figure 10 - Total sulfur-containing amino acid (TSAA) content of the wild type and transgenic tobacco lines. * Significant at $p<0.05$; ** Significant at $p<0.01$
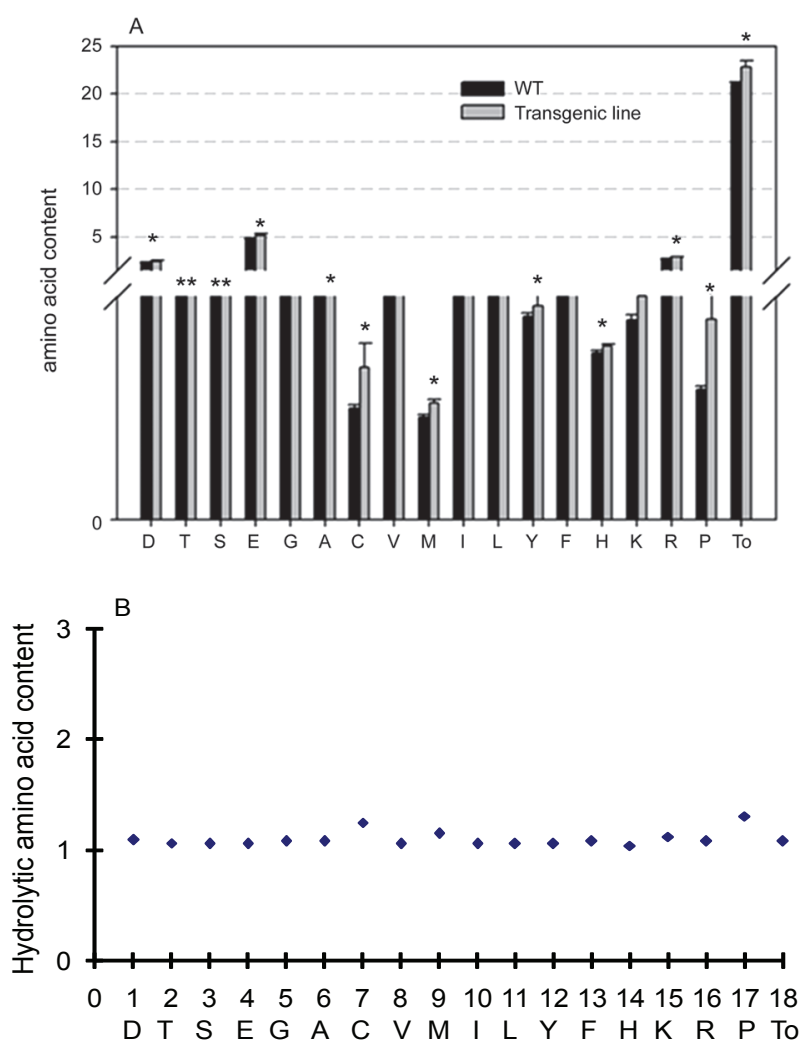

Figure 11 - Analysis of the amino acid content in the wild type and GmDof17-1 T1 transgenic tobacco line seeds. A. Histogram of 17 amino acid types in WT and transgenic lines. B. The $X$-axis shows the abbreviations for 17 amino acid types; the Y-axis depicts the ratio of the amino acid content of the transgenic line and wild type. Ratio $>1$, the transgenic lines have higher amino acid contents; ratio $<1$, the wild type has a higher amino acid content. To: total amino acid content. 
Casimiro, I.; Marchant, A.; Bhalerao, R.P.; Beeckman, T.; Dhooge, S.; Swarup, R.; Graham, N.; Inzé, D.; Sandberg, G.; Casero, P.J.; Bennett, M. 2001. Auxin transport promotes Arabidopsis lateral root initiation. The Plant Cell 13: 843-852.

Chen, H.; He, H.; Yu, D. 2010. Over expression of a novel soybean gene modulating $\mathrm{Na}^{+}$and $\mathrm{K}^{+}$transport enhances salt tolerance in transgenic tobacco plants. Physiologia Plantarum 141: 11-18.

De Paolis, A.; Sabatini, S.; De Pascalis, L.; Costantino, P.; Caopne, I. 1996. A rolB regulatory factor belongs to a new class of single zinc finger plant proteins. The Plant Journal 10: 215-223.

Esaka, M.; Fujisawa, K.; Goto, M.; Kisu, Y. 1992. Regulation of ascorbate oxidase expression in pumpkin by auxin and copper. Plant Physiology 100: 231-237.

Falkenberg, B.; Witt, I.; Zanor, M.I.; Steinhauser, D.; MuellerRoeber, B.; Hesse, H.; Hoefgen, R. 2008. Transcription factors relevant to auxin signalling coordinate broad-spectrum metabolic shifts including sulphur metabolism. Journal of Experimental Botany 59: 2831-2846.

Gabriele, S.; Rizza, A.; Martone, J.; Circelli, P.; Costantino, P.; Vittorioso, P. 2010. The Dof protein DAG1 mediates PIL5 activity on seed germination by negatively regulating GA biosynthetic gene AtGA3ox1. The Plant Journal 61: 312-323.

Genoud, T.; Metraux, J.P. 1999. Crosstalk in plant cell signaling: structure and function of the genetic network. Trends in Plant Science 4: 503-507.

Gutierrez, L.; Wuytswinkel, O.V.; Castelain, M.; Bellini, C. 2007. Combined networks regulating seed maturation. Trends in Plant Science 12: 294-300.

Kang, H.G.; Foley, R.C.; Onate-Sanchez, L.; Lin, C.G.T.; Singh, K.B. 2003. Target genes for OBP3, a Dof transcription factor, include novel basic helix-loop-helix domain proteins inducible by salicylic acid. The Plant Journal 35: 362-372.

Kang, H.G.; Singh, K.B. 2001. Characterization of salicylic acidresponsive, arabidopsis Dof domain proteins: over expression of OBP3 leads to growth defects. The Plant Journal 21: 329339.

Kepinski, S. 2006. Integrating hormone signaling and patterning mechanisms in plant development. Current Opinion in Plant Biology 9: 28-34

Kisu, Y.; Ono, T.; Shimofurutani, N.; Suzuki, M.; Esaka, M. 1998. Characterization and expression of a new class of zinc finger protein that binds to silencer region of Ascorbate Oxidase gene. Plant Cell Physiology 39: 1054-1064.

Kumar, S.; Nei, M.; Dudley, J.; Tamura, K. 2008. MEGA: A biologist-centric software for evolutionary analysis of DNA and protein sequences. Briefings in Bioinformatics 9: 299-306.

Livak, K.J.; Schmittgen, T.D. 2001. Analysis of relative gene expression data using real-time quantitative PCR and the $2^{-\triangle \triangle C T}$ method. Methods 25: 402-408.

Mena, M.; Vicente-Carbajosa, J.; Schmidt, R.J.; Carbonero, P. 1998. An endosperm-specific Dof protein from barley, highly conserved in wheat, binds to and activates transcription from the prolamin-box of a native B-hordein promoter in barley endosperm. The Plant Journal 16: 53-62.

Plesch, G.; Ehrhardt, T.; Mueller-Roeber, B. 2001. Involvement of TAAAG elements suggests a role for Dof transcription factors in guard cell-specific gene expression. The Plant Journal 28: 455-464.
Quint, M.; Gray, W.M. 2006. Auxin signaling. Current Opinion in Plant Biology 9: 448-453.

Ravanel, S.; Gakiere, B.; Job, D.; Douce, R. 1998. The specific features of methionine biosynthesis and metabolism in plants. Proceedings of the National Academy of Sciences 95: 7805-7812.

Shan, X.; Yan, J.; Xie, D. 2012. Comparison of phytohormone signaling mechanisms. Current Opinion in Plant Biology 15: 84-91.

Stamm, P.; Kumar, P.P. 2010. The phytohormone signal network regulating elongation growth during shade avoidance. Journal of Experimental Botany 61: 2889-2903.

Tian, A.G.; Wang, J.; Cui, P. 2004. Characterization of soybean genomic features by analysis of its expressed sequence tags. Theoretical and Applied Genetics 108: 903-913.

Vicente-Carbajosa, J.; Moose, S.P.; Parsons, R.L.; Schmidt, R.J. 1997. A maize zinc-finger protein binds the prolamin box in zein gene promoters and interacts with the basic leucine zipper transcriptional activator Opaque 2. Proceedings of the National Academy of Sciences 94: 7685-7690.

Wang, H.W.; Zhang, B.; Hao, Y.J.; Huang, J.; Tian, A.G.; Liao, Y.; Zhang, J.S.; Chen, S.Y. 2007. The soybean Dof-type transcription factor genes, GmDof4 and GmDof11, enhance lipid content in the seeds of transgenic Arabidopsis plants. The Plant Journal 52: 716-729.

Ward, J.W.; Cufr, C.A.; Denze, M.A.; Neff, M.M. 2005. The Dof transcription factor OBP3 modulates phytochrome and cryptochrome signaling in Arabidopsis. The Plant Cell 17: 475485.

Washio, K. 2001. Identification of Dof proteins with implication in the gibberellin-regulated expression of a peptidase gene following the germination of rice grains. Biochimica et Biophysica ActaBiomembranes http://www.sciencedirect.com/science/article/ pii/S0167478101002512 - CORR1\#CORR1 1520: 54-62.

Weising, K.; Atkinson, R.G.; Gardner, R.C. 1995. Genomic fingerprinting by microsatellite-primed PCR: a critical evaluation. PCR Methods and Applications 4: 249-255.

Xin, B.; Chen, X.Y.; Ouyang, K.X.; Pian R.Q.; Li, W. 2009. Isolation and sequence analysis of a Dof protein gene (PtDof1) in populus. Forestry Studies in China 11: 93-98.

Yanagisawa, S.; Izui, K. 1993. Molecular cloning of two DNAbinding proteins of maize that are structurally different but interact with the same sequence motif. Journal of Biological Chemistry 268: 16028-16036.

Yanagisawa, S. 2002. The Dof family of plant transcription factors. Trends in Plant Science 7: 555-560.

Yanagisawa, S. 2004. Dof domain proteins: plant-specific transcription factors associated with diverse phenomena unique to plants. Plant and Cell Physiology 45: 386-391.

Yanagisawa, S.; Sheen, J. 1998. Involvement of maize Dof zinc finger proteins in tissue-specific and light-regulated gene expression. The Plant Cell 10: 75-89.

Yang, J.; Yang, M.F.; Wang, D.; Chen, F.; Shen, S.H. 2010. JcDof1, a Dof transcription factor gene, is associated with the lightmediated circadian clock in Jatropha curcas. Physiologia Plantarum 139: 324-334.

Yu, J.J.; Han, S.Y.; Shi, G.X.; Yu, D.Y. 2012. Characterization of a novel curled-cotyledons mutant in soybean [Glycine max (L.) Merr.]. African Journal of Biotechnology 11: 14889-14898. 